\title{
Statine im präventiven Einsatz
}

Der Zusammenhang zwischen Statintherapie und Leberkrebsrisiko bei Hepatitis$\mathrm{C}$-Virus (HCV)-Infizierten ist bereits in mehreren Studien untersucht worden. Danach reduzieren Statine das Leberkrebsrisiko um 25-40\%. Erstmals haben nun taiwanesische Ärzte diesen Zusammenhang in einer bevölkerungsgestützten Kohortenstudie bei unterschiedlichen Statindosierungen überprüft. Von 261.000 HCV-infizierten Krankenversicherten erkrankten 28.000 in 2,8 Millionen Personenjahren an einem hepatozellulären Karzinom. Von den mehr als 35.000 Patienten mit HCV-Infektion, die mit einem Statin in einer kumulativen Tagesdosis pro Jahr (cDDD) von über 28 behandelt wurden, erkrankten knapp 1.400 an Leberkrebs. Unter den HCV-Patienten ohne Statintherapie (cDDD < 28) - das waren fast 226.000 Infizierte - wurde bei mehr als 26.500 Patienten ein he- patozelluläres Karzinom diagnostiziert. Im Vergleich zur Gruppe der Patienten ohne Statintherapie war das relative Risiko für Leberkrebs bei einer cDDD zwischen 28 und 89 pro Jahr um $34 \%$, bei einer cDDD zwischen 90 und 180 pro Jahr um $53 \%$ und bei einer cDDD über 180 pro Jahr um $67 \%$ reduziert. Damit habe die Statintherapie einen ähnlich präventiven Effekt wie eine Interferontherapie, so die Ärzte. Aus der Studie gehe auch hervor, dass die Statintherapie bei HCVInfizierten sicher sei. Allerdings sei zu bedenken, dass die gleichzeitige Applikation der Statine Atorvastatin, Lovastatin und Simvastatin mit den HCV-Proteasehemmern Boceprevir oder Telaprevir die Wahrscheinlichkeit erhöhe, an einer Myopathie oder einer Rhabdomyolyse zu erkranken.

Für eine generelle Empfehlung von Statinen zur Prävention von Leberkrebs ist

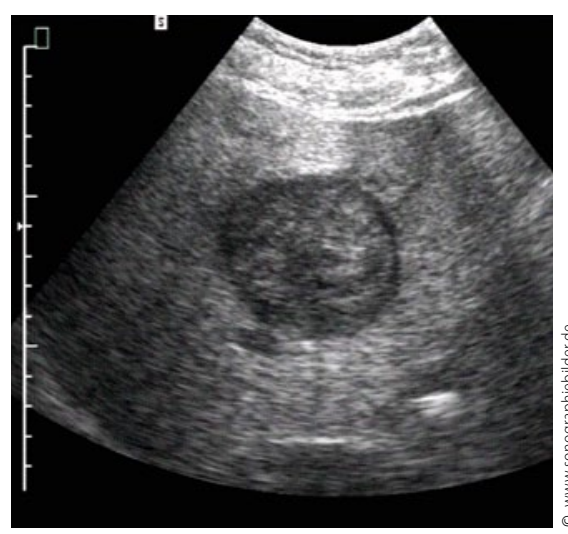

Häufige Spätfolge einer Hepatitis: Hepatozelluläres Karzinom (HCC).

es nach Ansicht der Onkologin Abby B. Siegel vom Columbia University Medical Center in New York noch zu früh. Für sie ist auch die Zeit noch nicht reif für eine randomisierte Präventionsstudie. Unter anderem müssten zuvor sowohl der antikarzinogene Effekt von Statinen als auch deren Wirkung auf die HCV-Aktivität genauer erforscht werden. Peter Leiner

Tsan YT et al. Statins and the Risk of Hepatocellular Carcinoma in Patients With Hepatitis C Virus Infection. J Clin Oncol. 2013 Mar 18. [Epub ahead of print]

\section{Auch nach Fatigue, Angst und Depression fahnden}

Fatigue ist bei Krebserkrankungen nicht nur ein kurzzeitiges Problem. Bei Darmkrebspatienten wiesen niederländische Forscher noch Jahre nach der Diagnose ein deutlich erhöhtes Fatigue-Risiko nach. Von über 3.700 Patienten mit kolorektalem Kar-

\section{kurz notiert}

\section{Leukämieforscher heben Biomarker-Schatz}

Die EU unterstützt ein internationales Forschungsprojekt zur personalisierten Krebsmedizin mit insgesamt 5,5 Millionen Euro. Ulmer Forscher sind mit einem Volumen von 450.000 Euro daran beteiligt, heißt es in einer Pressemitteilung der Universität UIm. Ziel ist, aus der Vielzahl der Biomarker bei Leukämien die relevanten herauszufiltern und valide, schnelle Tests für sie zu entwickeln bzw. erprobte Tests zu optimieren. zinom (CRC) litten in der Studie fast $40 \%$ unter Fatigue-Symptomen. Die Betroffenen berichteten vor allem, dass sie schnell ermüdeten und Probleme hätten, klare Gedanken zu fassen. Die höchsten Werte in der 50-Punkte umfassenden Skala FAS (Fatigue Assessment Scale) hatten Patienten, bei denen weniger als fünf Jahre seit Diagnosestellung vergangen waren; sie kamen auf durchschnittlich 21 Punkte (höhere Werte stehen für schwerere Symptome). Patienten, bei denen die Diagnose bereits fünf oder mehr Jahre zurücklag, erreichten im Durchschnitt immer noch 20 Punkte.

In einer Vergleichsgruppe von 338 repräsentativen Niederländern, die an einer Online-Befragung teilgenommen hatten, war die Fatigue-Prävalenz mit $22 \%$ zwar ebenfalls relativ hoch, das Risiko bei den CRC-Patienten war jedoch signifikant größer ( $<<0,0001)$.

Bei operierten Patienten war eine anschließende Radiochemotherapie ein un- abhängiger Risikofaktor für die Entwicklung einer Fatigue (OR 1,63). Bei Angst und depressiven Symptomen erhöhte sich das Fatigue-Risiko ebenfalls, und zwar um den Faktor 1,16 bzw. 1,38. Jeder fünfte Krebspatient wies nach dem HAD-Score (Hospital Anxiety and Depression) eine klinisch relevante Angst oder Depression auf.

Von den Patienten, die in den ersten fünf Jahren in dem Selbstauskunftsbogen SCQ (Self-Administered Comorbidity Questionnaire) über depressive Symptome berichteten, hätten allerdings nur $57 \%$ eine antidepressive Therapie erhalten, kritisieren die Autoren. In der Vergleichsgruppe wurden dagegen insgesamt $75 \%$ behandelt.

Darmkrebspatienten müssen stärker auf mögliche Langzeitfolgen einer Chemotherapie hingewiesen werden, folgern die Autoren. Vor allem auf die psychischen Probleme dieser Patienten müsse stärker eingegangen werden. Elke Oberhofer

Thong MSY et al. Quantifying fatigue in (longterm) colorectal cancer survivors: A study from the population-based Patient Reported Outcomes Following Initial treatment and Long term Evaluation of Survivorship registry. Eur J Cancer. 2013 Feb 28. [Epub ahead of print] 